\title{
Die Summe der Einzelaspekte und das Gesamtbild
}

D ass die Summe der Einzelbetrachtungen nicht dem Gesamtbild entspricht, ist eine relativ triviale Erfahrung mit dennoch großer Tragweite. Deutlich wird dies gerade in der Entwicklung moderner Gesundheitssysteme, denn das Endergebnis der Betrachtungen wird nach den im Vordergrund stehenden, wissenschaftlich, wirtschaftlich, ärztlich oder ethisch geprägten persönlichen Standpunkten „gefärbt.“ Dieser Erkenntnis kann man sich leider nicht verschließen, wenn moderne Entwicklungen im Gesundheitswesen analysiert werden. Ein Beispiel ist die immer hochdifferenziertere wissenschaftliche Erforschung biologischer Systeme, wobei ein Erkenntnisgewinn in der Tat nur durch Spezialisierung und Differenzierung möglich scheint. Bei der Anwendung und Wertung wissenschaftlicher Fortschritte für den Menschen ist allerdings vonnöten, das allgemeine Ziel, nämlich die Behandlung unserer Patienten, nicht aus dem Auge zu verlieren.

Die angedeuteten Zusammenhänge sind im Besonderen bei den expandierenden Möglichkeiten der Diagnostik zu beobachten. Denn deren zum Teil spektakuläre Einzelerkenntnisse verstellen leicht den Blick dafür, ob sich die Methoden auch für die Anwendung am Menschen eignen. Gerade vor dem Hintergrund der Entwicklung molekularbiologischer Verfahren, sowohl in der allgemeinen Diagnostik als auch in prädiktiven Verfahren zur Vermeidung von Krankheiten, ist die Leistungsfähigkeit nur auf die praktische Medizin zu transferieren, wenn es gelingt, diese einzelnen Entwicklungen in den Gesamtkontext der praktischen Diagnostik zu integrieren.

Ohne die biologischen Rahmenbedingungen der menschlichen Existenz zu präzisieren (erinnert sei an den Ernährungszustand, den Hydratationszustand, tages- und jahreszeitliche Varianten), sind immer differenziertere und in der Regel kostenaufwändigere Diagnostiken sinnlos oder gar irreführend, da die Interpretation der gewonnenen Einzeldaten im Bezug auf das Hauptziel der Behandlung kranker Menschen nicht möglich ist. So mag ein hochbetagter Patient bei seiner Einlieferung aufgrund eines Verwirrtheitszustandes in die Notaufnahme einer Klinik ausnahmslos physiologische, biochemische Messergebnisse bei den laboratoriumsdiagnostischen Untersuchungen zeigen. Diese liefern jedoch erst dann zielführende Erkenntnisse, wenn ergründet wird, ob sie deshalb in physiologischen Konzentrationsbereichen gefunden werden, weil gleichzeitig eine beispielsweise durch einen Wassermangel verursachte massive Exsikkose Werte bedingt, die den tatsächlichen Krankheitszustand nicht repräsentieren.

Die ärztliche und gesellschaftspolitische Bewertung gesundheitsrelevanter Maßnahmen muss die Maximen des ärztlichen Handelns mit den zugrunde liegenden ökonomischen Konsequenzen und Gegebenheiten in Einklang bringen. Dem einleitenden Kapitel eines Buches der Heidelberger Gruppe Kulozik, Hentze, Hagemeier und Bartram „Molekulare Medizin“ kann man nur zustimmen, wenn die Autoren konstatieren, „dass das Vorliegen einer vom Arzt festzustellenden medizinischen Indikation, wie etwa die Anwendung spezifischer diagnostischer und therapeutischer Verfahren, die Grundlage ärztlichen Handelns bleiben muss" - auch zur Bewertung der Verwendung vorhandener finanzieller Ressourcen. Das schließt natürlich auch ein, dass in die moderne Ausbildung der Ärzte diese neuen, speziellen Erkenntnisse und Techniken integriert werden müssen, die ja in den bisherigen Curricula nur unzulänglich abgebildet werden.

Durch die modernen Entwicklungen ergibt sich eine ungeheuere Erweiterung der Bandbreite diagnostischer und therapeutischer Verfahren, bei der sich vernachlässigte Gebiete der medizinischen Ausbildung in stärkerem Maße als bisher auswirken. Dazu zählt beispielsweise der Umgang mit dem Patienten in strukturierten, zielorientierten und unter Zeitdruck stehenden Gesprächen zur sinnvollen Anwendung kostenintensiver hochdifferenzierter moderner Verfahren. Ohne Zweifel ist ohne Grundlagenwissen in den Biowissenschaften die zukünftige Bewertung solcher Verfahren auch im Sinne der ökonomischen Absicherung nicht möglich.

Der klinikarzt will in seinem interdisziplinären Ansatz mit Abhandlungen von Schwerpunktthemen diese Notwendigkeit erkennbar machen, um sicherzustellen, dass einerseits die hochleistungsfähigen neuen Erkenntnisse in den Grundlagen der Lebenswissenschaften umgesetzt werden können, und auf der anderen Seite durch eine Rückbesinnung auf das Hauptziel der Behandlung kranker Menschen das Gesamtbild durch diese Einzelschwerpunkte auch ökonomisch nicht aus dem Gleichgewicht gerät.

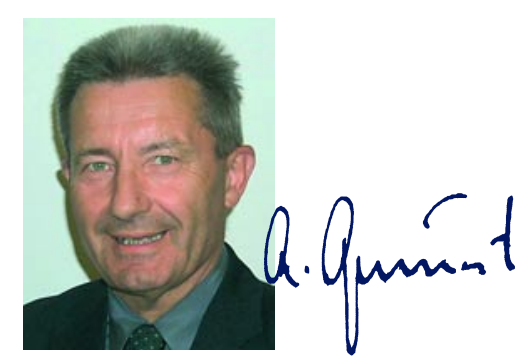

Prof. Dr. Dr. Dr. A. Grünert, Ulm 